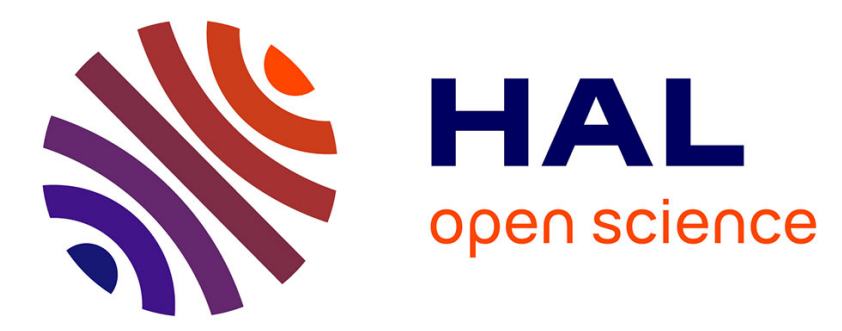

\title{
Determination of Equilibrium Association Constants of Ligand-DNA Complexes by Electrospray Mass Spectrometry
}

\author{
Valérie Gabelica
}

\section{- To cite this version:}

Valérie Gabelica. Determination of Equilibrium Association Constants of Ligand-DNA Complexes by Electrospray Mass Spectrometry. Methods in Molecular Biology, 2009, pp.89-101. 10.1007/978-160327-418-0_6. hal-01524124

HAL Id: hal-01524124

https://hal.science/hal-01524124

Submitted on 17 May 2017

HAL is a multi-disciplinary open access archive for the deposit and dissemination of scientific research documents, whether they are published or not. The documents may come from teaching and research institutions in France or abroad, or from public or private research centers.
L'archive ouverte pluridisciplinaire HAL, est destinée au dépôt et à la diffusion de documents scientifiques de niveau recherche, publiés ou non, émanant des établissements d'enseignement et de recherche français ou étrangers, des laboratoires publics ou privés. 


\title{
Determination of equilibrium association constants of ligand-DNA complexes by electrospray mass spectrometry
}

\author{
Valérie Gabelica ${ }^{1,2}$ \\ ${ }^{1}$ Physical Chemistry and Mass Spectrometry Laboratory, Department of Chemistry, \\ University of Liège, Belgium. \\ ${ }^{2}$ GIGA-Systems Biology and Chemical Biology, University of Liège, Belgium. \\ v.gabelica@ulg.ac.be
}

\begin{abstract}
Electrospray mass spectrometry can be used to detect ligand-DNA noncovalent complexes formed in solution. This chapter describes how to determine equilibrium association constants of the complexes. Particular attention is devoted to describing how to tune an electrospray mass spectrometer using a 12-mer oligodeoxynucleotides duplex in order to perform these experiments. This protocol can then be applied to any nucleic acid structure that can be ionized with electrospray mass spectrometry.
\end{abstract}

Key Words: Mass Spectrometry, Electrospray, Nucleic acids, Oligonucleotides, Duplex, GQuadruplex, Ligand, Equilibrium constants.

\section{Introduction}

The introduction of electrospray sources was a major breakthrough in the field of biomolecule mass spectrometry $(1 ; 2)$. Electrospray allows producing intact ions directly from large biomolecules directly from a solution injected in a capillary (3). The softness of electrospray (the absence of fragmentation thanks to the limited energy given by thermal heating and collisional activation) is such that even noncovalent complexes can be detected intact. This is also the case for DNA and RNA noncovalent complexes (higher-order structures, nucleic acid complexes with small molecules or with proteins), as reviewed in references (4-8).

In order to obtain soft source conditions that are suitable to maintain complexes intact, it is necessary to change some instrumental parameters compared to those typically used for covalent, less fragile analytes. Instead of giving the parameters that were optimized for our 
instruments, we will describe a tuning procedure that can be applied to any electrospray mass spectrometer (Section 3.2).

Peaks in the mass spectra are characterized by their $\mathrm{m} / \mathrm{z}$ and their area. The masses of the peaks give immediate information on the stoichiometry of all complexes formed simultaneously in the solution, while in spectrophotometry methods the stoichiometry is deduced by fitting of ligand binding curves. In addition, the relative peak areas in the mass spectra are proportional to the relative concentrations of the corresponding species in solution:

$$
\frac{[X]}{[Y]}=R \times \frac{A_{(X)}}{A(Y)}
$$

The easiest approximation is to consider that $R=1$, i.e. that the ratio between peak areas in the mass spectra are equal to the ratio between the concentrations of the corresponding species in solution. This is valid only if species $\mathrm{X}$ and $\mathrm{Y}$ under consideration have similar masses, shapes, hydrophobicity, and the ions produced have the same total charge.

For nucleic acid-ligand complexes, it means that $\mathrm{R}=1$ is valid for small molecules binding to a nucleic acid target without changing dramatically the target's conformation. The ideal case is that of minor groove binders of DNA duplexes (9). As there is currently no easy and widely applicable method to determine the value of $\mathrm{R}$, all equations described here are based on the hypothesis that $\mathrm{R}=1$.

However, the protocol and equations can be applied to any nucleic acid structure that can be ionized with electrospray mass spectrometry, even if the validity of the $\mathrm{R}=1$ hypothesis is not known. In general, deviation from that ideal behavior manifests itself by the fact that the ratio between peak areas can depend on the charge state of the ions in the mass spectra. Therefore, in order to estimate the error due to the hypothesis, we describe a procedure involving determination of the association constants for the different charge states.

\section{Materials}

\subsection{Sample preparation}

1. $40 \mathrm{nmol}$ dCGCGAATTCGCG,$+40 \mathrm{nmol}$ of each synthetic oligonucleotide you want to include in your assay. We typically use OliGold® oligonucleotides from Eurogentec (www.eurogentec.com), with standard purification "process OliGold". (Note 1). Dissolve all lyophilized sequences to $400 \mu \mathrm{M}$ in bi-distilled water, make $100 \mu \mathrm{L}$ aliquots and store at $-20^{\circ} \mathrm{C}$.

2. Ligand to be tested, as a powder or as a stock solution of concentration $\geq 50 \mu \mathrm{M}$ (Note 2). 
3. Water, $\mathrm{Na}$ and $\mathrm{K}<1 \mathrm{mg} / \mathrm{kg}$ (Note 3). We typically use deionized water that is further distilled twice in house.

4. Methanol, $\mathrm{Na}$ and $\mathrm{K}<1 \mathrm{mg} / \mathrm{kg}$ (Note 3), e.g. puriss. p.a., absolute, ACS reagent, $\geq 99.8 \%$ (65543, Fluka, www.sigmaaldrich.com).

5. Ammonium acetate $\left(\mathrm{NH}_{4} \mathrm{OAc}\right)$ solution Bioultra, for molecular biology, $5 \mathrm{M}$ in $\mathrm{H}_{2} \mathrm{O}$ (09691, Fluka, www.sigmaaldrich.com) (Note 3).

6. Microcon ${ }^{\circledR}$ YM-3 (cut-off $=3 \mathrm{kDa}$, yellow) Centrifugal Filter Units (Millipore, www.millipore.com).

7. Store all solvents and sample solutions in polypropylene tubes (Note 4).

\subsection{Electrospray mass spectrometer}

1. In section 3.2, we will describe a tuning procedure that can be applied to different mass spectrometers equipped with an electrospray source (Note 5). In addition, we will provide indicative source tuning parameters for four instruments, from three different manufacturers.

a. Finnigan LCQ classic (Thermo, San Jose, CA, USA) with standard ESI source block.

b. Micromass Q-TOF II (now Waters, Manchester, UK) with standard Z-spray source.

c. Waters Q-TOF Ultima Global (Waters, Manchester, UK) with dual MALDI/ESI Z-spray source.

d. Bruker Apex-Qe 9.4T FTICR-MS (Bruker Daltonics, Bremen, Germany) with the Bruker Apollo 1 source.

2. A clean $250 \mu \mathrm{L}$ syringe and a syringe pump capable of delivering flow rates of 1-10 $\mu \mathrm{L} / \mathrm{min}$.

\section{Methods}

The methods will be described in the case of ligand binding to double-stranded DNA. The general procedure is also applicable to other oligonucleotide structures like intramolecular folds, triplex DNA, or G-quadruplexes. For G-quadruplexes, the criteria used for tuning the instrumental parameters are different from the duplexes because G-quadruplexes embed a certain amount of specific cations. Specific variations of the protocol for G-quadruplexes are described in the notes section. 


\subsection{Sample preparation}

1. If not known precisely, determine the strand concentration by UV absorption spectrophotometry at $260 \mathrm{~nm}$, using extinction coefficients calculated from http://biophysics.idtdna.com/.

2. Prepare $400 \mu \mathrm{L}$ of $50 \mu \mathrm{M}$ duplex [dCGCGAATTCGCG] $]_{2}$ in $150 \mathrm{mM} \mathrm{NH}_{4} \mathrm{OAc}$ by mixing $100 \mu \mathrm{L}$ stock solution ( $400 \mu \mathrm{M}$ in single strand) with $300 \mu \mathrm{L} \mathrm{NH}_{4} \mathrm{OAc} 200$ $\mathrm{mM}$. Heat the solution in simmering water bath for 5 minutes, allow cooling to room temperature. Store at $4{ }^{\circ} \mathrm{C}$.

3. Prepare stock solutions for each synthetic oligonucleotide you want to include in your assay, final concentration $50 \mu \mathrm{M}$ in the desired structure (e.g. $50 \mu \mathrm{M}\left[\mathrm{TG}_{4} \mathrm{~T}\right]_{4}$ requires $200 \mu \mathrm{M}$ single strand) (Note 6).

4. To prepare oligonucleotide solutions ready to inject in the mass spectrometer: mix (in that order) $5 \mu \mathrm{L}$ oligonucleotide $50 \mu \mathrm{M}$ with $35 \mu \mathrm{L}$ aqueous ammonium acetate of the desired concentration, then add $10 \mu \mathrm{L}$ methanol (Note 7). Final concentration is $5 \mu \mathrm{M}$ oligonucleotide and $20 \%$ methanol.

5. Oligonucleotide-ligand mixtures ready to inject in the mass spectrometer are $5 \mu \mathrm{M}$ oligonucleotide, the desired concentration of ligand (typically 5 or $10 \mu \mathrm{M}$ ), and $20 \%$ methanol. If the ligand stock was in methanol, take that amount into account in the total $20 \%$.

6. If mass spectra reveal it necessary to remove sodium or potassium from an oligonucleotide sample:

a. If not known, determine the extinction coefficient of the folded oligonucleotide structure at room temperature (this extinction coefficient is not the same as for unfolded single strand). For duplex DNA, use http://biophysics.idtdna.com/.

b. Place $\mathrm{X} \mu \mathrm{L}$ of the solution to be desalted on a Microcon ${ }^{\circledR} \mathrm{YM}-3$ filter, centrifuge to near dryness.

c. Add $400 \mu \mathrm{L}$ of $150 \mathrm{mM} \mathrm{NH}_{4} \mathrm{OAc}$ in the filter, and centrifuge to near dryness (repeat three times).

d. Add $\mathrm{X} \mu \mathrm{L}$ of $\mathrm{NH}_{4} \mathrm{OAc}$ of the desired concentration to re-dilute the oligonucleotide and homogenize.

e. Place the filter upside down in a fresh Microcon vial, and centrifuge to get the oligonucleotide solution in the vial. 
f. Oligonucleotide concentration has changed during that process. Determine the final concentration with UV spectrophotometry by using the extinction coefficient determined in (a).

\subsection{Instrument tuning}

1. Switch the mass spectrometer to negative ion mode (Note 8).

2. Calibrate the instrument in negative ion mode according to the manufacturer's instructions.

3. Beware that optimal tuning parameters can vary from instrument to instrument, even from the same manufacturer. However, tuning parameters for a given machine remain constant over time if the instrument is kept clean, and can be re-used later. If you already have tuning parameters for DNA complexes using your instrument, go to section 3.3.

4. If it is the first time you tune your mass spectrometer for DNA complexes, start with the following parameters:

a. Finnigan LCQ classic (Thermo, San Jose, CA, USA) with standard ESI source block. Sheath gas flow rate $($ arb. $)=60$, Aux gas flow rate $($ arb. $)=5$, I Spray voltage $(\mathrm{kV})=3.7$, Capillary temperature $\left({ }^{\circ} \mathrm{C}\right)=180$, Capillary voltage $=-10$ $\mathrm{V}$, Tube lens offset $=35$. Multipole 1 offset $=4$, Lens voltage $(\mathrm{V})=30$, Multipole 2 offset $(V)=8$, Multipole RF amplitude $\left(V_{p-p}\right)=600$.

b. Micromass Q-TOF II (now Waters, Manchester, UK) with Z-spray source. Spray voltage $=2.2 \mathrm{kV}$, Cone voltage $=20 \mathrm{~V}$, Source block temperature $=$ $70{ }^{\circ} \mathrm{C}$, Desolvation temperature $=80^{\circ} \mathrm{C}$, collision energy $=10 \mathrm{~V}$, ion energy $=$ 1.8 .

c. Waters Q-TOF Ultima Global (Waters, Manchester, UK) with dual MALDI/ESI Z-spray source. First set the source pressure at $3.8 \mathrm{mbar}$ (as measured on the pirani gauge). Spray voltage $=2.2 \mathrm{kV}$, Cone voltage $=$ $100 \mathrm{~V}$, RF Lens 1 voltage $=60 \mathrm{~V}$, Source temperature $=70^{\circ} \mathrm{C}$, Desolvation temperature $=80^{\circ} \mathrm{C}$, collision energy $=10 \mathrm{~V}$, ion energy $=1.8$.

d. Bruker Apex-Qe 9.4T FTICR-MS (Bruker Daltonics, Bremen, Germany) with Apollo 1 source. Capillary $=3400 \mathrm{~V}$, End plate $=2900 \mathrm{~V}$, CapExit $=-45 \mathrm{~V}$, Skimmer1 $=-30 \mathrm{~V}$, Skimmer2 $=-4 \mathrm{~V}$, Offset $=-2.5 \mathrm{~V}$, DryGasTemp $=$ $150{ }^{\circ} \mathrm{C}$, ColCellTrap $=5 \mathrm{~V}$.

e. For instruments not described here, start from any negative ion parameters, and be prepared to lower all voltages and temperatures significantly.

5. Inject a sample of duplex [dCGCGAATTCGCG $]_{2}$ using a syringe pump at $4 \mu \mathrm{L} / \mathrm{min}$. To find which of your instrumental parameters are critical for studying the complexes, 
change one parameter at a time and observe the ratio between the peak corresponding to the duplex ${ }^{5-}$ (monoisotopic mass $=1456.8516$, average mass $\left.=1457.5421\right)$ and the peak corresponding to the single strand ${ }^{3-}$ (monoisotopic mass $=1213.8751$, average mass $=1214.4587)$ (Note 9). On the four instruments described here, the most important parameters are:

a. Finnigan LCQ classic (Thermo, San Jose, CA, USA) with standard ESI source block: Capillary temperature, Capillary voltage, and Tube lens offset.

b. Micromass Q-TOF II (now Waters, Manchester, UK) with Z-spray source: Cone voltage. Collision energy of $10 \mathrm{~V}$ is recommended by the manufacturer, but for very fragile complexes you may have to lower this voltage down to $2 \mathrm{~V}$.

c. Waters Q-TOF Ultima Global (Waters, Manchester, UK) with dual MALDI/ESI Z-spray source: source pressure and RF Lens 1. Collision energy: see Q-TOF II.

d. Bruker Apex-Qe 9.4T FTICR-MS (Bruker Daltonics, Bremen, Germany) with Apollo 1 source: CapExit and Skimmer1.

6. In doing so, you have probably observed that softer conditions are obtained at the expense of sensitivity. To decide the final values of parameters, observe the relative intensity of the duplex ${ }^{5-}$ peak and its first ammonium adduct (monoisotopic mass = 1460.2568, average mass $=1460.9481)($ Note 10). Chose instrumental parameters that give an intensity of first ammonium adduct peak between 20 and $30 \%$ of the intensity of the free duplex ${ }^{5-}$. Store these parameters.

\subsection{Recording electrospray mass spectra for the DNA-ligand complexes}

1. Using the instrumental parameters determined previously, record a mass spectrum for a sample containing $5 \mu \mathrm{M}$ of DNA, without ligand, injected at $4 \mu \mathrm{L} / \mathrm{min}$. Try to assign all major peaks. You should observe your intact DNA at different charge states. You may also observe the separate constituents (single strands). The different $\mathrm{m} / \mathrm{z}$ are given by $\mathrm{m} / \mathrm{z}=\left(m_{\mathrm{DNA}}-\mathrm{z}\right) / \mathrm{z}$, where $m_{\mathrm{DNA}}$ is the mass of the neutral DNA in Daltons (Note 11), and $z$ is the charge state.

2. Fine tune the instrument for your particular oligonucleotide. For each intense charge state (signal-to-noise ratio $>20$ ), find source parameters at which the intensity of the first ammonium adduct peak (found at $\left.\mathrm{m} / \mathrm{z}=\left(m_{\mathrm{DNA}}+17-\mathrm{z}\right) / \mathrm{z}\right)$ is about $20 \%$ of the adduct-free peak (Note 12). Note down these parameters. If your oligonucleotide is a G-quadruplex, see Note 13. For troubleshooting, see Note 14. 
3. Sodium adducts are found at $m / z=\left(m_{\mathrm{DNA}}+n \times 22-z\right) / z$. If the first sodium adduct peak is larger than $30 \%$ of the no-adduct peak, desalt the oligonucleotide sample as described in 3.1.6., then repeat steps 1 and 2. (Note 15)

4. Inject a sample containing $5 \mu \mathrm{M}$ DNA and $5 \mu \mathrm{M}$ ligand, at a flow rate $\leq 4 \mu \mathrm{L} / \mathrm{min}$ (Note 16). If the ligand binds to the nucleic acid, for each charge state you see additional peaks at $m / z=\left(m_{\mathrm{DNA}}+n \times m_{\text {ligand }}-z\right) / z$, where $n$ is the number of ligands bound, and $m_{\text {ligand }}$ is the mass of the "neutral" ligand (Note 17).

5. Record separate mass spectra at different voltages: one optimal voltage for each abundant charge state.

6. Wash the electrospray source capillaries with $1 \mathrm{~mL}$ bi-distilled water to remove ligand and DNA.

7. Inject a sample containing $5 \mu \mathrm{M}$ DNA and $10 \mu \mathrm{M}$ ligand (same DNA and ligand as in step 4). Record separate mass spectra at different voltages: one optimal voltage for each abundant charge state.

8. Wash the electrospray source capillaries with $1 \mathrm{~mL}$ bi-distilled water to remove ligand and DNA.

9. Before running another DNA-ligand mixture, test with DNA alone that complexes are not detectable any longer (Note 18). If you have a particular nucleic acid to which the ligand binds strongly, use that nucleic acid for this test.

10. Wash the electrospray source capillaries with $1 \mathrm{~mL}$ bi-distilled water to remove DNA.

11. To test another ligand with the same oligonucleotide, go back to step 4. To test another oligonucleotide, go back to step 1.

\subsection{Calculation of equilibrium association constant}

1. For all mass spectra recorded for DNA-ligand mixtures (total concentration of DNA = $C_{\mathrm{DNA}}=5 \mu \mathrm{M}$, total concentration of ligand $=C_{\mathrm{L}}$ ), annotate all peaks corresponding to $[1 \text { DNA }+n \text { Ligands }]^{z-}(n=0$ to $N)$. Determine the maximum number $N$ of ligands bound.

2. Substract the background from the mass spectra. Ensure that the peak shape is conserved.

3. For each charge state $z$, use the mass spectrum recorded at the optimum voltage for that charge state. Integrate (Notes 19, 20) the peaks of each $(1: n)^{z-}$ species $(n=0$ to $N)$, and write the peak areas $A_{(1: n)}$ in a worksheet. 
4. In the worksheet, for each charge state separately, calculate the concentration of free DNA (1:0) and each complex (1:n) as follows:

$$
[1: n]=C_{D N A} \times \frac{A_{(1 \cdot n)}}{\sum_{n=0}^{N} A_{(1: n)}}
$$

5. For each charge state separately, calculate the concentration of free ligand using the mass balance equation (Note 21):

$$
[\mathrm{L}]=\mathrm{C}_{\mathrm{L}}-\sum_{\mathrm{n}=1}^{\mathrm{N}} \mathrm{n} \times[1: \mathrm{n}]
$$

6. Now you have the concentrations of all free species in solution, you can calculate, for each charge state and for each mixture the stepwise equilibrium association (Ka,n) and dissociation $(\mathrm{Kd}, \mathrm{n})$ constants:

$\mathrm{K}_{\mathrm{a}, \mathrm{n}}=\frac{1}{\mathrm{~K}_{\mathrm{d}, \mathrm{n}}}=\frac{[1: \mathrm{n}]}{[\mathrm{L}][1:(\mathrm{n}-1)]}$

7. For a given DNA target and a given ligand, examine the constants determined for each ligand concentration (at least 2). If the constants determined at different ligand concentrations do not agree with each other, repeat the mass spectrometry experiment, including sample preparation. If the discrepancy persists, see Note 21.

8. Constants determined from different charge states may differ. This is an intrinsic limitation of the method, due to the fact that relative intensities do not exactly reflect relative concentrations in solution. Reject outliers only if based on valid statistical tests. Calculate the mean value of the constant and the standard deviation. This standard deviation gives the uncertainty on the absolute value of the constant due to the electrospray mass spectrometry method.

\section{Notes}

1. The Oligold $®$ purification process is a Reverse-Phase (RP) purification. The RPcartridge devices used by Eurogentec are stable up to $\mathrm{pH}=13$, so the ammonium hydroxide solution after synthesis, diluted in water, is loaded directly on the packing. It is the level of desalting attained by this purification process that makes Oligold $\mathbb{R}$ oligonucleotides particularly suitable for mass spectrometry experiments.

2. What solvent can I use if my ligand is not water-soluble? Methanol. If not soluble in methanol either, prepare a concentrated solution in DMSO and then dilute with 
methanol. The final DMSO concentration in the solution injected in the electrospray source must be $\leq 0.5 \%$.

3. Sodium and potassium must be avoided, because during electrospray the solvent droplets evaporate, and positive ions around the negatively charged DNA condense around the DNA. This leads to a distribution of species [DNA $+\mathrm{n} \mathrm{Na} / \mathrm{K}$ ] that is heterogeneous in mass, and this translates in the mass spectra into a distribution of peaks. This alters the sensitivity of the method (total intensity is distributed over several peaks) and even the mass accuracy (if the peak without $\mathrm{Na} / \mathrm{K}$ is not identified). A substitute salt is nevertheless needed in order to obtain the desired ionic strength. Ammonium acetate is the salt of choice because both ammonium and acetate are "volatile" in the sense that ammonium ions condensing around a phosphate will give a proton to the phosphate and leave as ammonia $\left(\mathrm{NH}_{3}\right)$. Similarly, acetate anions can abstract protons and leave as neutral acetic acid. In electrospray mass spectrometry, $\mathrm{NH}_{4} \mathrm{OAc}$ can be used at concentrations up to $150 \mathrm{mM}$, corresponding to physiological ionic strength. Finally, ammonium cations are also capable of stabilizing G-quadruplexes by insertion in between G-tetrads.

4. Glassware can be contaminated with $\mathrm{NaCl}$ and $\mathrm{KCl}$ that is not removed by autoclaves. Also beware that some low quality polystyrene tubes may contain traces of polyethylene glycol that is solubilized by methanol, and gives undesirable peaks separated by $44 \mathrm{Da}$ in the mass spectra.

5. Can nanospray be used instead of electrospray? Nanospray capillaries are thin glass capillaries terminated by a very thin needle. It is possible to spray DNA-ligand complexes using nanospray, but three difficulties can be encountered. (1) Glass capillaries can be contaminated by $\mathrm{Na} / \mathrm{K}$ salts. If so, use quartz capillaries. (2) Thin capillaries clog rapidly when using $150 \mathrm{mM} \mathrm{NH}_{4} \mathrm{OAc}$ and results are not always reproducible quantitatively. With nanospray, it is recommended to use capillaries with reproducible tip opening (pre-opened tip) and to repeat the measurement at least three times to test the reproducibility of relative intensities.

6. Assays can be performed in any ammonium acetate concentration from 0 to $150 \mathrm{mM}$. Important: $\mathrm{NH}_{4} \mathrm{OAc}$ has no buffering capacities. It is an ampholyte (acid of one couple and base of another couple in equal quantities), hence the $\mathrm{pH}$ is the mean of the pKa's (7.0). Use acetic acid to acidify your solution, or ammonium hydroxide basify your solution, if so desired.

7. By adding some methanol to the sample, the surface tension of the mixture is lower than in pure water. This makes droplet evaporation more favorable, resulting in a higher electrospray signal. You can test this on your own instrument by comparing the same sample with $0 \%, 10 \%$ and $20 \%$ methanol. If the instrument is sensitive enough to work in $0 \%$ or $10 \%$ methanol, you can of course do so. However, although they were performed in $20 \%$ methanol, association constants determined by electrospray mass spectrometry were found in good agreement with those determined by other 
methods. It is reported that up to $50 \%$ methanol does not perturb DNA conformation (10), and that aliphatic alcohols even stabilize G-quadruplex DNA (11). In any case, always add methanol just before injection into the mass spectrometer (do not store DNA solutions in methanol).

8. Although it is possible to obtain mass spectra in positive ion mode, the relative intensities do not reflect the relative abundances in solution (12). Equilibrium association constants can be determined only in the negative ion mode.

9. In general, decreasing acceleration voltages and decreasing temperatures leads to softer conditions, i.e. less destruction of the complexes, and higher duplex/single strand ratio. Important: some parameters expressed in volts are not directly related to the voltages applied in the instrument (for example, in Thermo/Finnigan instruments, the tube lens offset voltage is an offset applied to a ramp of voltages that itself depends on the mass range chosen; the more positive the tube lens offset, the softer the conditions). Finally, increasing the pressures inside the instrument can have different effects depending on instrument design (for example, increasing the source pressure in Waters instruments leads to softer conditions, but increasing the pressure inside the collision cell leads to harder conditions). If you don't know the instrument design in detail, tune all these parameters empirically.

10. Do not confound ammonium adduct (mass difference of $+17 / z$ ) and sodium adduct (mass difference of $+22 / z$ ) or potassium adduct (mass difference of $+38 / z$ ).

Ammonium adducts are removed (see Note 3 ) when source conditions get harsher, while sodium and potassium adducts are not.

11. The mass of the neutral DNA is calculated by assuming that all phosphates are neutralized by protons.

12. At this stage, change only one source voltage that allows you to go from very soft conditions (low voltage) to hard conditions (high voltage). On the instruments described here, these are: (a) on the LCQ, the capillary voltage; (b) on standard Zspray sources, the cone voltage; (c) on the dual Z-spray source of the Q-TOF Ultima Global, the RF Lens 1 voltage; (d) on the Apollo Source the CapExit voltage. This optimum voltage can be the same for all charge states, but most often you will find the optimum voltage is lower for higher charge states, and increases as the charge state increases. If you see no ammonium adducts even at the lowest voltages allowed on your instrument, lower the source temperatures.

13. Tuning the instrument in the case of G-quadruplexes is different, because Gquadruplexes prepared in $\mathrm{NH}_{4} \mathrm{OAc}$ incorporate a specific number of ammonium cations in between their G-tetrads. In general, a quadruplex containing $t$ consecutive G-tetrads incorporates $(t-1)$ ammoniums, and the theoretical $\mathrm{m} / \mathrm{z}$ of the intact quadruplex is given by: $m / z=\left(m_{\mathrm{DNA}}+(t-1) \times 17-z\right) / z$. In practice, a distribution of ammonium adducts is observed (from 0 if all inner ammoniums are removed, to $>t-1$ 
if nonspecific adducts on the phosphates are formed in addition to the specific adducts between the tetrads). For each charge state, tune the mass spectrometer to have the highest relative intensity for the ( $t-1)$ adduct. Note that this can be easy for some Gquadruplexes and very difficult for others (13).

14. Troubleshooting if you have peaks you cannot assign. Check if these can correspond to a shorter sequence (for example one base is missing); in that case have the DNA resynthesized. Check if these can be due to backbone fragmentation of your oligonucleotide in the mass spectrometer (you can compute the most common fragments for any sequence with the Mongo Oligo Mass Calculator v2.06 freeware: http://library.med.utah.edu/masspec/mongo.htm); in that case use softer source conditions. Check if these can correspond to unanticipated higher-order structures (several nucleic acids associating). Finally, check if this could correspond to ammonium, sodium, potassium adducts, adducts with your calibrant,...

15. If sodium adducts persist even when using clean solvents and desalting the best you can, you will have to take the intensity of all sodium adduct peaks into account in the calculation of the equilibrium association constants. It is more tedious and less precise than with a well-desalted sample, but the determination of the constants is still feasible. Ease of desalting is one of the reason we typically use DNA targets no longer than 30 bases: shorter oligonucleotides minimize the number of sodium binding sites.

16. Using low ( $5 \mu \mathrm{M}$ total DNA) concentration and low ( $4 \mu \mathrm{L} / \mathrm{min})$ flow rate ensures that the production of ions is not limited by the surface concentration of charges available on the droplets compared to the concentration of analytes in the droplets (14). This ensures that all forms of DNA (free and complexed) have equal chances to be ionized.

17. Even if the ligand is not neutral in the experimental conditions, the mass of the "neutral" ligand is used in this formula because the total charge of the complex is already accounted for in " $z$ ". To calculate the mass of the "neutral" ligand, for a given ligand structure, subtract $i$ Daltons (i protons) if your ligand is a cation of charge $i+$, or add $j$ Daltons ( $j$ protons) if your ligand is an anion of charge $j$-. In fact, in the charged complex, if a ligand is charged, protonation changes must occur on the phosphates in order for the total charge to remain the same.

18. Some ligands may stick to capillaries. If the ligand is more soluble in DMSO than in water, wash the capillaries as follows: first with $1 \mathrm{~mL} \mathrm{DMSO}$, then $500 \mu \mathrm{L}$ methanol, then $500 \mu \mathrm{L}$ water. Because of the possible remanence of ligands, when testing several ligand concentrations it is recommended to conduct tests from lower concentration to higher concentration. When testing the binding of several ligands to a given target, design the test so as to alternate ligands of significantly different masses, in order to detect such remanence, and carry out washing if needed.

19. Peak areas are proportional to the ion current. However if all peaks have exactly the same shape, which is usually the case when the signal-to-noise ratio is high, peak 
heights can be used instead of peak areas in the calculation of peak ratios. In principle, the whole ammonium/sodium adducts distribution of each $(1: n)^{z_{-}}$complex must be summed up in calculating the area of the $(1: n)^{z-}$ complex. In practice, if the shape of the adduct distribution is exactly the same for $n=0$ to $N$, then the area of one representative peak can be used in the calculation of the peak ratios. Increasing the signal-to-noise ratio and smoothing the mass spectra help obtaining more similar peak shapes. For example, smoothing the isotope distribution is useful to avoid integrating each isotopic peak and summing them up afterwards.

20. Remember that a peak $(1: n)^{z_{-}^{-}}$can be quantified only if its signal-to-noise ratio is $\geq 10$. This sets the sensitivity limit of the method, and the order of magnitude of association constants that can be determined. With very large association constants the signal of free DNA can be impossible to quantify. If so, run experiments with lower total concentrations. With very low association constants the signal of the complexes can be impossible to quantify. If so, run experiments with higher ligand concentration. In all cases, as the signal-to-noise increases with the number of scans summed up, you can always improve your sensitivity by recording spectra for a longer time if you have enough sample solution.

21. If the calculated [L] is negative the association constant cannot be determined. If you find a negative free ligand concentration, this is often due to an error in ligand concentration, either in the stock solution (the concentration of the stock ligand solution cannot be determined precisely when weighting small quantities). Repeat the experiment including the preparation of a new stock solution of ligand. If this happens again, another possibility is that for the system under study the complexes respond in electrospray much better than the free nucleic acid, and that the intensities are not proportional to the concentrations (see Introduction).

\section{Acknowledgements}

I would like to thank Frédéric Rosu, Nicolas Smargiasso, Joëlle Widart, Dominique Baiwir, and Edwin De Pauw for their comments on the manuscript. The Fonds de la Recherche Scientifique-FNRS and the University of Liège are gratefully acknowledged for financial support. The author is a FNRS research associate.

\section{References}

1. Whitehouse,M., Dreyer,R.N., Yamashita,M. and Fenn,J.B. (1985) Electrospray interface for liquid chromatographs and mass spectrometers. Anal. Chem., 57, 675679.

2. Fenn,J.B., Mann,M., Meng,C.K., Wong,S.F. and Whitehouse,C.M. (1989) Electrospray ionization for mass spectrometry. Science, 246, 64-71. 
3. Fenn,J.B. (2003) Electrospray wings for molecular elephants (Nobel lecture). Angew. Chem. Int. Ed Engl., 42, 3871-3894.

4. Hofstadler,S.A. and Griffey,R.H. (2001) Analysis of noncovalent complexes of DNA and RNA by mass spectrometry. Chem. Rev., 101, 377-390.

5. Beck,J., Colgrave,M.L., Ralph,S.F. and Sheil,M.M. (2001) Electrospray ionization mass spectrometry of oligonucleotide complexes with drugs, metals, and proteins. Mass Spectrom. Rev., 20, 61-87.

6. Banoub,J.H., Newton,R.P., Esmans,E., Ewing,D.F. and Mackenzie,G. (2005) Recent developments in mass spectrometry for the characterization of nucleosides, nucleotides, oligonucleotides, and nucleic acids. Chem. Rev., 105, 1869-1916.

7. Hofstadler,S.A. and Sannes-Lowery,K.A. (2006) Applications of ESI-MS in drug discovery: interrogation of noncovalent complexes. Nature Rev. Drug Discov., 5, 585595.

8. Rosu,F., De Pauw,E. and Gabelica,V. (2008) Electrospray mass spectrometry to study drug-nucleic acid interactions. Biochimie, 90, 1074-1087.

9. Gabelica,V., Galic,N., Rosu,F., Houssier,C. and De Pauw,E. (2003) Influence of response factors on determining equilibrium association constants of non-covalent complexes by electrospray ionization mass spectrometry. J. Mass Spectrom., 38, 491501.

10. Mel'nikov,S.M., Khan,M.O., Lindman,B. and Jönsson,B. (1999) Phase behavior of single DNA in mixed solvents. J. Am. Chem. Soc., 1130-1136.

11. Smirnov,I.V. and Shafer,R.H. (2007) Electrostatics dominate quadruplex stability. Biopolymers, 85, 91-101.

12. Rosu,F., Pirotte,S., De Pauw,E. and Gabelica,V. (2006) Positive and negative ion mode ESI-MS and MS/MS for studying drug-DNA complexes. Int. J. Mass Spectrom., 253, 156-171.

13. Rosu,F., Gabelica,V., Houssier,C., Colson,P. and De Pauw,E. (2002) Triplex and quadruplex DNA structures studied by electrospray mass spectrometry. Rapid Commun. Mass Spectrom., 16, 1729-1736.

14. Kuprowski,M.C. and Konermann,L. (2007) Signal response of coexisting protein conformers in electrospray mass spectrometry. Anal. Chem., 79, 2499-2506. 